\title{
MicroRNA-212 targets FOXA1 and suppresses the proliferation and invasion of intrahepatic cholangiocarcinoma cells
}

\author{
LEI ZHU, FEIZHOU HUANG, GANG DENG, WANPIN NIE, WEI HUANG, \\ HONGBO XU, SHAOPENG ZHENG, ZHONGJIE YI and TAO WAN
}

\begin{abstract}
Department of Hepatobiliary and Pancreatic Surgery, The Third Xiangya Hospital of Central South University, Changsha, Hunan 410013, P.R. China
\end{abstract}

Received June 11, 2015; Accepted June 20, 2016

DOI: $10.3892 /$ etm.2016.3824

\begin{abstract}
MicroRNAs (miRNAs), which are a class of small RNAs, have been shown to negatively regulate the expression of their target genes by directly binding to the 3'-untranslated region (3'-UTR) of mRNA. miRNA dysregulation has been associated with the pathogenesis of numerous types of human cancer. However, the role of miRNAs in intrahepatic cholangiocarcinoma (ICC) has yet to be fully elucidated. The present study aimed to investigate the role of miR-212 in the growth and metastasis of ICC in vitro, as well as the underlying
\end{abstract} mechanism. Reverse transcription-quantitative polymerase chain reaction (RT-qPCR) and western blotting were used to examine mRNA and protein expression. An MTT assay and transwell assay were conducted to determine cell proliferation and invasion rates. The results of the RT-qPCR demonstrated that miR-212 was downregulated in the majority of invest gated ICC tissues, as compared with their matched adjacent non-tumor tissues. In addition, miR-212 expression was shown to be markedly downregulated in three ICC cell lines, as compared with human intrahepatic biliary epithelial cells. Furthermore, restoration of miR-212 expression significantly suppressed the protiferation and invasion of ICC QBC939 cells. Forkhead box protein A1 (FOXA1) was predicted to be a putative target of miR-212 by bioinformatics analysis with TargetScan. Therefore, a luciferase reporter assay was conducted to confirm that miR-212 was able to directly bind to the 3'-UTR of FOXA1 mRNA. In addition, using western blot analysis, the protein expression of FOXA1 was shown to be negatively regulated by miR-212 in ICC QBC939 cells. In

Correspondence to: Dr Gang Deng, Department of Hepatobiliary and Pancreatic Surgery, The Third Xiangya Hospital of Central South University, 138 Tongzipo Road, Changsha, Hunan 410013, P.R. China

E-mail: doctordenggang@163.com

Key words: intrahepatic cholangiocarcinoma, microRNA, forkhead box protein A1, proliferation, invasion conclusion, it was demonstrated that FOXA1 was frequently upregulated in various ICC tissues and cell lines. The results of the present study suggested that miR-212 inhibits the proliferation and invasion of ICC cells by directly targeting FOXA4, and thus may be considered a potential candidate for the treatment of IC

\section{Introduction}

Intrahepatie cholangiocarcinoma (ICC) is the second most common primary liver cancer (1), and the most common neoplasm of the biliary tract (2). Due to its increasing incidence worldwide and relatively poor prognosis, ICC remains an often fatal disease (3). Therefore, elucidating the molecular mechanisms underlying ICC is required in order to develop novel therapeutic strategies for ICC.

MicroRNAs (miRNAs) are a class of endogenous small RNAs containing 22 nucleotides that have been reported to be frequently dysregulated in various types of human cancer $(4,5)$. By negatively regulating the protein expression levels of their target genes, miRNAs have key roles in cancer progression $(6,7)$. Since miRNAs may act as oncogenes or tumor suppressors, they may be considered as potential molecular targets or candidates for the treatment of various human cancer types $(8,9)$. Among the tumor-associated miRNAs, miR-212 has been shown to serve a suppressive role in human cancer (10). For instance, upregulation of miR-212 is closely associated with a poor prognosis in patients with esophageal cancer (11). In addition, Jiang et al (12) demonstrated that overexpression of the miR-212/132 cluster inhibited the proliferation of human lung cancer cells. Liang et al (13) also reported that miR-212 acts as a tumor suppressor in hepatocellular carcinoma. In particular, the authors demonstrated that overexpression of miR-212 resulted in the suppression of $\mathrm{H} 3 \mathrm{~K} 4$ demethylase retinoblastoma binding protein 2 expression, inhibition of cell proliferation and induction of cellular senescence in hepatocellular carcinoma cells (13). Conversely, another study reported that miR-212 promoted pancreatic cancer cell growth and invasion by targeting the hedgehog signaling pathway receptor patched-1 (14). Therefore, miR-212 may serve a dual role in cancer, and its role in 
different cancer types requires further investigation. To the best of our knowledge, the exact role of miR-212 in ICC has yet to be elucidated.

The present study aimed to investigate the expression levels of miR-212 in ICC tissues and matched normal adjacent tissues. Furthermore, the current study examined the role of miR-212 in the regulation of ICC cell proliferation and invasion, as well as the underlying regulatory mechanism of its action.

\section{Materials and methods}

Tissue collection. The present study was approved by the Ethics Committee of the Third Xiangya Hospital of Central South University (Changsha, China). ICC tissues and their matched adjacent non-tumor tissues were collected from 15 patients with ICC at the Third Xiangya Hospital of Central South University between October 2013 and June 2014. The ICC patients included 9 males and 6 females, with an age range of 48-74 years (mean age, 62.7 years). ICC was diagnosed with histopathological examination performed by a pathologist ( 3 patients at disease stage II, 8 at stage III and 4 at stage IV). The patients did not receive any treatment prior to surgery. Written informed consent was obtained from all patients. All tissue samples were snap-frozen in liquid nitrogen following surgical removal and stored at $-80^{\circ} \mathrm{C}$ until further use.

Cell culture. Human QBC939 ICC cell lines and human intrahepatic biliary epithelial cells (HIBEpiC) were purchased from the Cell Bank of the Chinese Academy of Sciences (Shanghai, China). Cells were cultured in Dulbecco's Modified Eagle Medium (DMEM; Thermo Fisher Scientific, Inc. Waltham, MA, USA) supplemented with $10 \%$ fetal bovine serum (FBS; Thermo Fisher Scientific, Inc.) and incubated at $37^{\circ} \mathrm{C}$ in an atmosphere with $5 \%$

Transfection. MISSION ${ }^{\circledR}$ miR-212 mimics (cat. no.HMI0377) and MISSION ${ }^{\circledR}$ negative control (miR-NC; cat. no.HMC0002) were provided by Sigma-Aldrich (St. Lotis, MO, USA). The cells were seeded in 24-well plates (100,000 cells/well) and transfected with $100 \mathrm{nM}$ miR-212 mimics or miR-NC using Lipofectamine 2000 reagent (Invitrogen; Thermo Fisher Scientific, Inc.). The cells were then cultured in an incubator at $37^{\circ} \mathrm{C}$ for $48 \mathrm{~h}$, for use during reverse transcription-quantitative polymerase chain reaction (RT-qPCR) and western blot analysis.

$R T$ - $q P C R$. Following tissue homogenization and centrifugation $\left(12,000 \mathrm{x} \mathrm{g}, 10 \mathrm{~min}, 4^{\circ} \mathrm{C}\right)$, total RNA was extracted from the fresh tissues or cells using TRIzol reagent (Invitrogen; Thermo Fisher Scientific, Inc.), according to the manufacturer's protocol. The purity and concentration of the RNA was determined using a Thermo Nanodrop 2000 spectrophotometer (Thermo Fisher Scientific, Inc.). The miR-212 expression levels were determined using the TaqMan ${ }^{\circledR}$ MicroRNA Reverse Transcription kit (cat. no. 4366597; Applied Biosystems; Thermo Fisher Scientific, Inc.), according to the manufacturer's protocol. U6 was used as a normalization control for miRNA expression. For the detection of the mRNA expression levels, $0.5 \mu \mathrm{g}$ RNA was reverse transcribed to cDNA using the RevertAid First Strand cDNA Synthesis kit (cat. no. K1621; Thermo Fisher Scientific, Inc.). Glyceraldehyde 3-phosphate dehydrogenase (GAPDH) was used as a normalization control for gene expression. A negative control used $\mathrm{H}_{2} \mathrm{O}$ instead of cDNA. The PCR reaction was performed on an Applied Biosystems ${ }^{\circledR} 7500$ Real-Time PCR system (Thermo Fisher Scientific, Inc.). The PCR mixture included $0.33 \mu \mathrm{l}$ cDNA solution, $10 \mu \mathrm{l}$ of $1 \mathrm{X}$ TaqMan universal PCR master mix, $2 \mu \mathrm{l}$ of $1 \mathrm{X}$ gene specific primer/probe set (all from Applied Biosystems; Thermo Fisher Scientific, Inc.), and $7.67 \mu 1 \mathrm{H}_{2} \mathrm{O}$ was added to obtain a final reaction volume of $20 \mu \mathrm{l}$. The primer sequences were as follows: FOXA1 forward, 5'-GCAATACTCGCCTTACGG CT-3' and reverse, 5'-TACACACCTTGGTAGTACGCC-3'; and GAPDH forward, 5'-CTGGGCTACACTGAGCACC-3' and reverse, 5'-AAGTGGTCGTTGAGGGCAATG-3'. The PCR reaction conditions were as follows: Initialization at $95^{\circ} \mathrm{C}$ for $5 \mathrm{~min}$, followed by 40 cycles of denaturation at $95^{\circ} \mathrm{C}$ for $15 \mathrm{sec}$ and annealing/elongation at $60^{\circ} \mathrm{C}$ for $1 \mathrm{~min}$. The relative fold-changes of miR-212 andFOXA1 mRNA expression were calculated using the $2^{-\Delta \Delta C \mathrm{a}}$ method (15).

Western blot analysis. QBC939 cells were lysed using lysis buffer (Beyotime Institute of Biotechnology, Wuhan, China), and total protein $(20 \mu 1)$ was extracted. The protein concentration was determined using a Bicinchoninic Acid Protein Assay kit (Thermo Fisher Scientific, Inc.), according to the manufacturer's instructions. Subsequently, total protein was separated by $10 \%$ SDS-PAGE, followed by transfer to polyvinylidene fluoride membranes (EMD Millipore, Billerica, MA, USA). The membranes were blocked with phosphate-buffered saline (PBS) and $5 \%$ non-fat milk at $4^{\circ} \mathrm{C}$. The membranes were incubated overnight at $4^{\circ} \mathrm{C}$ with polyclonal rabbit anti-human FOXA1 antibody (1:800; cat. no. AV32630; Sigma-Aldrich) and polyclonal rabbit anti-human GAPDH antibody (1:800; cat. no. G9545; Sigma-Aldrich). After washing three times with PBS, the membrane was incubated with polyclonal horseradish peroxidase-conjugated goat anti-rabbit secondary antibody (1:5,000; cat. no. R4880; Sigma-Aldrich) and visualized using an enhanced chemiluminescence reagent (EMD Millipore). The results were quantified using ImageJ version 1.1 (National Institutes of Health, Bethesda, MA, USA).

MTT assay. MTT assays were performed to assess cell proliferation. QBC939 cells transfected with miR-212 mimics or miR-NC were seeded into 96 -well plates $\left(1 \times 10^{5}\right.$ cells/well) and cultured for $48 \mathrm{~h}$, after which $10 \mathrm{mg} / \mathrm{ml}$ MTT was added to the medium and the cells were incubated for a further $4 \mathrm{~h}$. Subsequently, the supernatant was removed by centrifugation $(1,000 \mathrm{x} \mathrm{g}, 5 \mathrm{~min}$, room temperature) and $100 \mu \mathrm{l}$ DMSO was added to each well for $30 \mathrm{~min}$ to dissolve the formazan product. The optical density (OD) was measured at $492 \mathrm{~nm}$ using an ELx808 plate reader (BioTek Instruments, Inc., Winooski, VT, USA). The relative cell proliferation was calculated using optical density values, with the value of the control group set at 1 .

Transwell assay. Transwell assays were conducted to investigate cell invasion. QBC939 cells (1x105 cells/well) 
transfected with miR-212 mimics or miR-NC were seeded into the top chamber of Matrigel-coated polyethylene terephthalate membrane, after which $10 \%$ FBS was added into the lower chamber. After culturing for $24 \mathrm{~h}$, QBC939 cells in the upper chamber were removed, and the cells in the lower chamber were stained with $0.1 \%$ crystal violet for $30 \mathrm{~min}$, followed by counting under an optical microscope (Olympus Corporation, Tokyo, Japan).

Bioinformatics analysis and luciferase reporter assay. The targets of miR-212 were predicted using TargetScan (http://www.targetscan.org/). "Human" was selected as the species, and "miR-212" was entered. To validate whether FOXA1 was a target gene of miR-212, a luciferase assay was performed using the pMIR-REPORT vector (Applied Biosystems; Thermo Fisher Scientific, Inc.) containing the wild-type (WT) or mutant (MT) 3'-untranslated region (3'-UTR) of FOXA1. Co-transfection was performed in 24 -well plates $\left(1 \times 10^{5}\right.$ cells/well) containing $200 \mu \mathrm{l}$ DMEM. Briefly, QBC939 cells were co-transfected with $100 \mathrm{nM}$ pMIR-REPORT vector containing WT or MT FOXA1 3'-UTR, and with miR-212 mimics or miR-NC for $48 \mathrm{~h}$. Subsequently, the luciferase activity was determined using the Dual-Luciferase Reporter Assay system (Promega Corporation, Madison, WI, USA), according to the manufacturer's protocol.

Statistical analysis. Data are presented as the mean \pm standard deviation. Significant differences among the groups were determined using one-way analysis of variance with SPSS version 17.0 software (SPSS, Inc., Chicago, $\mathrm{H}, \mathrm{USA}$ ). $\mathrm{P}<0.05$ was considered to indicate a statistically significant difference. All experiments were performed in triplicate.

\section{Results}
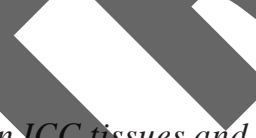

miR-212 expression is downregulated in ICC tissues and cell lines. In order to investigate the potential role of miR-212 in ICC, RT-qPCR was performed to determine the expression levels of miR-212 in ICC tissues and their matched adjacent non-tumor tissues. As shown in Fig. 1A, the expression levels of miR-212 were downregulated in the majority of ICC tissue samples, as compared with theirmatched adjacent tissues. The expression levels of miR-212 were upregulated in two stage II samples. In addition, miR-212 expression was significantly downregulated in ICC cell lines, as compared with HIBEpiC $(\mathrm{P}<0.05$; Fig. 1B). These results suggest that miR-212 may act as a tumor suppressor in ICC. Since miR-212 expression in QBC939 cells was decreased to a greater extent (Fig. 1B), this cell line was used in the subsequent experiments.

Restoration ofmiR-212 expressionsuppressesthe proliferation of ICC cells. The role of miR-212 in the regulation of ICC cell proliferation was investigated following transfection of human ICC QBC939 cells with miR-212 mimics or miR-NC. RT-qPCR was performed to determine the miR-212 levels in the cells following transfection. As is shown in Fig. 2A, the levels of miR-212 were significantly increased in QBC939 cells transfected with miR-212 mimics, as compared with the control group $(\mathrm{P}<0.05)$. MTT assay was also conducted to examine the cell proliferation capacity. As shown in Fig. 2B, ICC QBC939 cells overexpressing miR-212 demonstrated a significant decrease in cell proliferation, as compared with the control group $(\mathrm{P}<0.05)$. These results suggest that miR-212 has a suppressive effect on ICC cell proliferation.

Overexpression of miR-212 inhibits ICC cell invasion. ICC is typically associated with a high capacity for invasion and distal metastasis (1). Therefore, the role of miR-212 in the regulation of ICC cell invasion was investigated. As is shown in Fig. 3, overexpression of miR-212 significantly inhibited the invasion of ICC QBC939 cells, as compared with the control group $(\mathrm{P}<0.05$; Fig. 3). These results suggest that miR-212 has a suppressive effeet on ICC cell invasion.

FOXA1 is a target of $m i R-212$. In order to further investigate the molecular mechanisms underlying the involvement of miR-212 in ICC, the targets of miR-212 were analyzed using Targetscan. FOXA1 was predicted to be a putative target of miR-212, and their association was shown to be evolutionarily conserved (Fig. 4A). To validate this, a luciferase assay was performed, in which QBC939 cells were co-transfected with pMUR-REPQRT vector containing the WT or MT FOXA1 3'-UTR, and with miR-212 mimics or miR-NC (Fig. 4B). Transfection with miR-212 mimics significantly decreased the luciferase activity in the WT group, but not in the MT group (Fig. 4C). This suggests that miR-212 directly targets FOXA1 by binding to seed sequences in the 3'-UTR of FOXA1 mRNA. Furthermore, overexpression of miR-212 resulted in a significant decrease in the protein expression evels of FOXA1 in ICC QBC939 cells $(\mathrm{P}<0.05)$, although did not alter the mRNA expression level of FOXA1 Fig. 5A and B). These results suggest that miR-212 negatively regulates the expression of FOXA1 in ICC QBC939 cells at a post-transcriptional level.

FOXA1 is upregulated in the majority of ICC tissues and cell lines. The mRNA expression levels of FOXA1 were upregulated in the majority of ICC tissue samples, as compared with their matched normal adjacent liver tissues (Fig. 6A). Furthermore, the protein expression levels of FOXA1 were significantly upregulated in the ICC cell lines, as compared with HIBEpiC $(\mathrm{P}<0.05$; Fig. $6 \mathrm{~B})$. These results suggest that aberrant upregulation of FOXA1 may participate in the malignant progression of ICC.

\section{Discussion}

It has previously been demonstrated that miRNAs negatively regulate the expression of their target genes by binding to the 3'-UTR of their target mRNA (16). Furthermore, various miRNAs have been associated with the development and progression of numerous types of human cancer, including ICC (17-19). Tumor growth and metastasis are the two main characteristics of ICC that lead to a poor prognosis $(20,21)$. Therefore, understanding the role of miRNAs in these processes may aid in the diagnosis and treatment of ICC.

Dysregulation of miR-212 expression has been reported in several types of human cancer. For example, Park et al (22) demonstrated that miR-212 was upregulated in pancreatic 

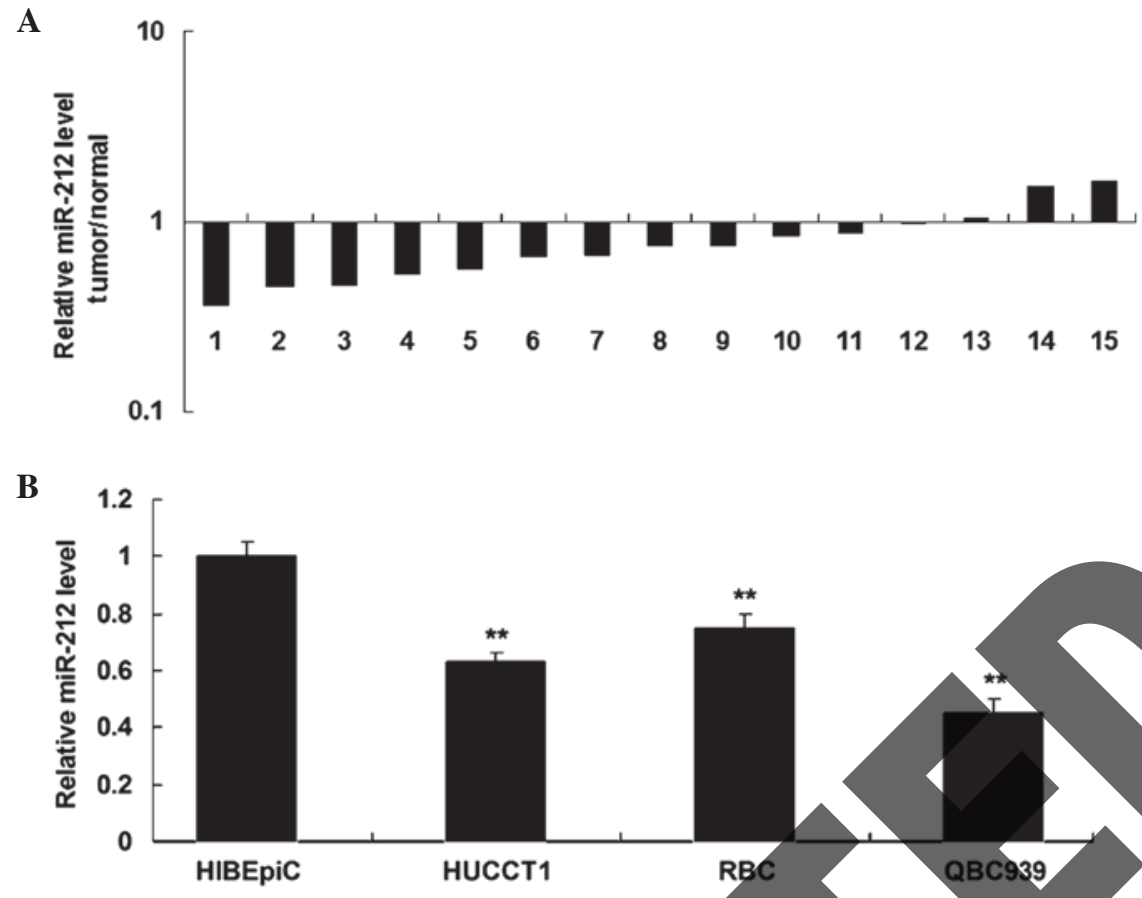

Figure 1. miR-212 expression levels in intrahepatic cholangiocarcinoma (ICC) tissues and cellythes. Reverse transcription-quantitative polymerase chain reaction was performed to determine the expression levels of miR-212 in (A) ICC tissues, as compared with then matched adjacent normal tissues, and (B) ICC cell lines, as compared with HIBEpiC. Data are presented as the mean \pm standard deviation. " $\mathrm{P}<0.05$ vs. HIBEpiC. miR-212, microRNA-212; HIBEpiC, human intrahepatic biliary epithelial cells.

adenocarcinoma tissues, and that knockdown of miR-212 reduced cell proliferation and induced a $\mathrm{G}_{2} / \mathrm{M}$ cell cycle arrest in Panc-1 cells. Furthermore, high expression levels of miR-212 were shown to predict a shorter overall survival, independent of age, gender, calendar year of surgery, KRAS mutation status, tumor stage, American Society of Anesthesiologists score, and localization and differentiation of pancreatic cancer (23). Similarly, it has been reported that miR-212 was upregulated in oral carcinoma (24). However, miR-212 has been shown to be frequently downregulated in several other types of human cancer. For instance, miR-212 was downregulated in gastric cancer and was shown to inhibit the DNA methylation by suppressing the expression of methyl-CpG-binding protein 2 (25). In addition, the expression of miR-212 was found to be downregulated in lung cancer, which may be due to histone modifications rather than DNA hypermethylation (26).

The present study revealed that miR-212 was downregulated in the majority of ICC tissues investigated, as compared with their matched adjacent non-tumor tissues, thus suggesting that miR-212 may act as a candidate tumor suppressor in the pathogenesis of ICC. Therefore, the ability of miR-212 to regulate ICC growth and metastasis in vitro was investigated in the current study. The results suggested that miR-212 significantly inhibited ICC cell proliferation and invasion, indicating a tumor suppressive role of miR-212 in ICC.

FOXA1, which is also known as HNF3 $\alpha$, encodes a member of the forkhead class of DNA-binding proteins, which are transcriptional activators of liver-specific genes, including albumin and transthyretin $(27,28)$. Furthermore, FOXA1 exhibited increased expression in liver development

and metabolism (29). Previous studies have reported that FOXA1 is involved in various human cancer types, including liver cancer (30-32). Zhao and Li (33) demonstrated that 

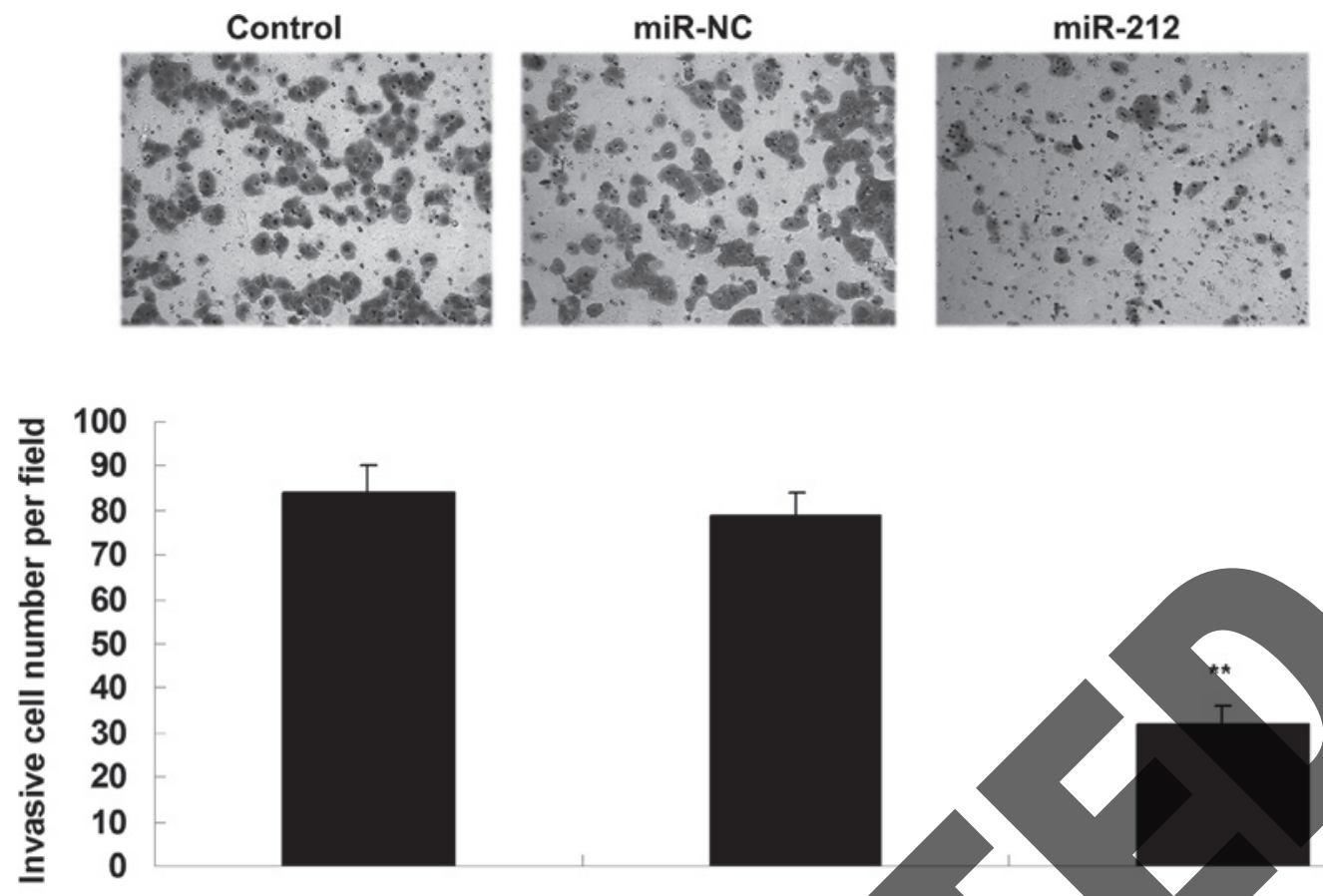

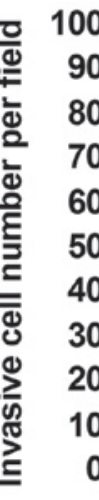

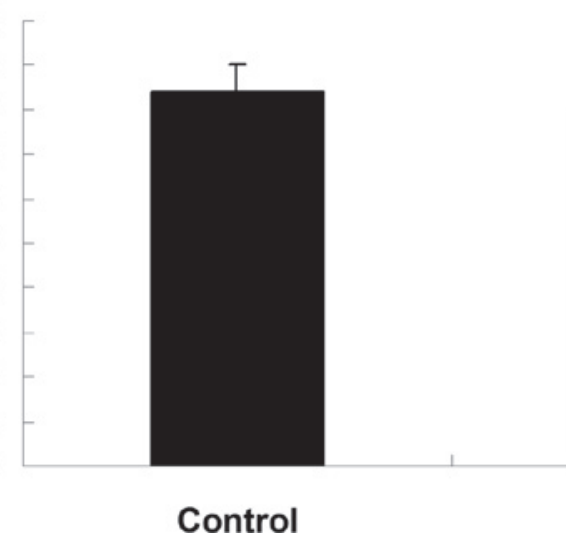

Figure 3. Transwell assays were conducted to assess the invasion of intrahepatic cholangiocarcinoma QBC939 cels transfected with miR-212 mimics or miR-NC. Non-transfected QBC939 cells were used as a control. Data are presented as the mean \pm standard deviation. " $\mathrm{P}<0.05$ vs. the control. miR-212, microRNA-212; miR-NC, microRNA-normal control.

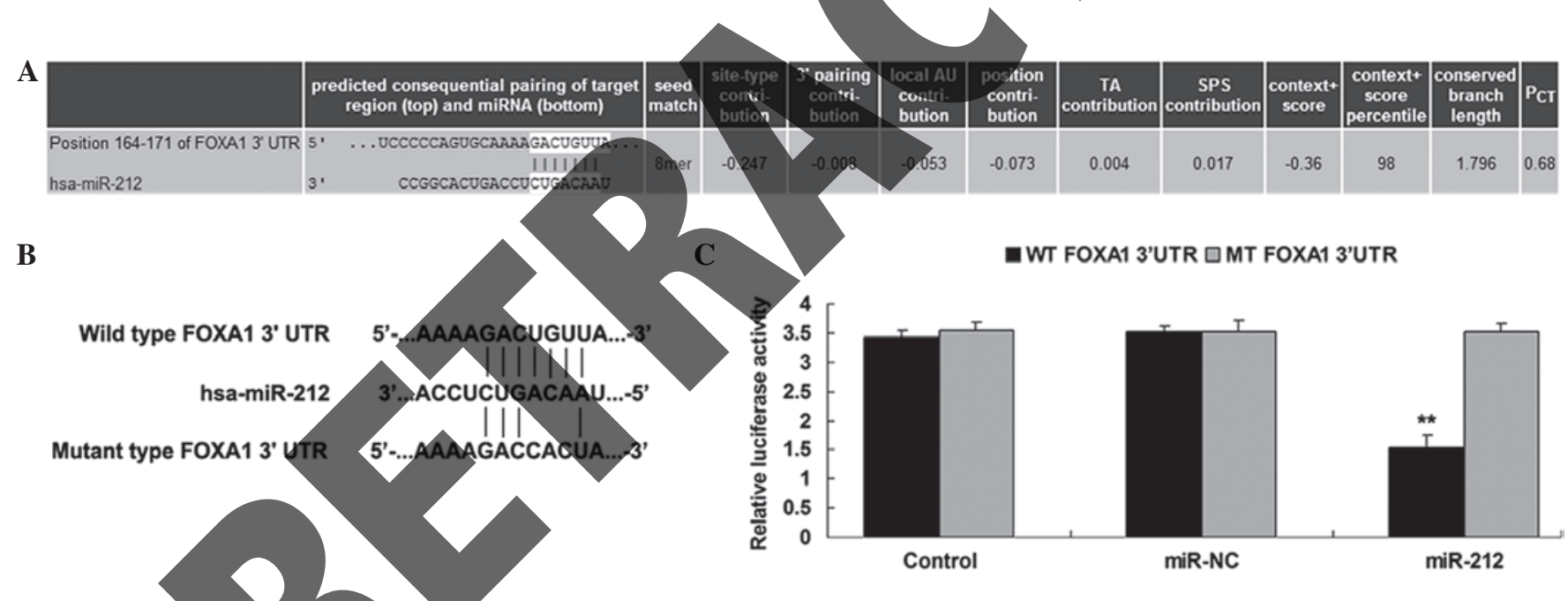

Figure 4. (A) Targetscan software predicted that FOXA1 was a putative target of miR-212. (B) The potential seed sequences of miR-212 within the wild-type and mutant 3'-UTR ofFOXA1 mRNA. (C) A luciferase assay was performed using the pMIR-REPORT vector containing the WT or MT FOXA1 3'-UTR, which were co-transfected into intrahepatic cholangiocarcinoma QBC939 cells with miR-212 mimics or miR-NC. In the control group, QBC939 cells were transfected with pMIR-REPORT vector containing WT or MT FOXA1 3'UTR only. Data are presented as the mean \pm standard deviation. ** P $<0.05$ vs. the control. miR-212, microRNA-212, 3'-UTR, 3'-untranslated region; FOXA1, forkhead box protein A1; WT, wild-type; MT, mutant; miR-NC, microRNA-normal control.

FOXA1 was essential for both estrogen and androgen signaling by acting as a central regulator of sexual dimorphism in liver cancer. However, the detailed role of FOXA1 in ICC remains unclear. The present study demonstrated that FOXA1 was upregulated in the majority of investigated ICC tissues and in ICC cell lines, as compared with their matched adjacent normal tissues and HIBEpiC, respectively. By performing bioinformatics analysis and a luciferase reporter assay, the present study demonstrated for the first time that FOXA1 was a direct target of miR-212 in ICC cells. In addition, FOXA1 was shown to be involved in miR-212-mediated
ICC cell proliferation and invasion. These results suggested that miR-212 suppresses ICC growth and metastasis by targeting FOXA1.

In conclusion, the present study demonstrated that miR-212 was aberrantly downregulated in ICC tissues and cell lines. Restoration of miR-212 expression significantly suppressed the proliferation and invasion of ICC cells by inhibiting the protein expression of FOXA1, a novel identified target of miR-212. The present study is the first to suggest that miR-212 may be a potential therapeutic candidate for ICC. 
A

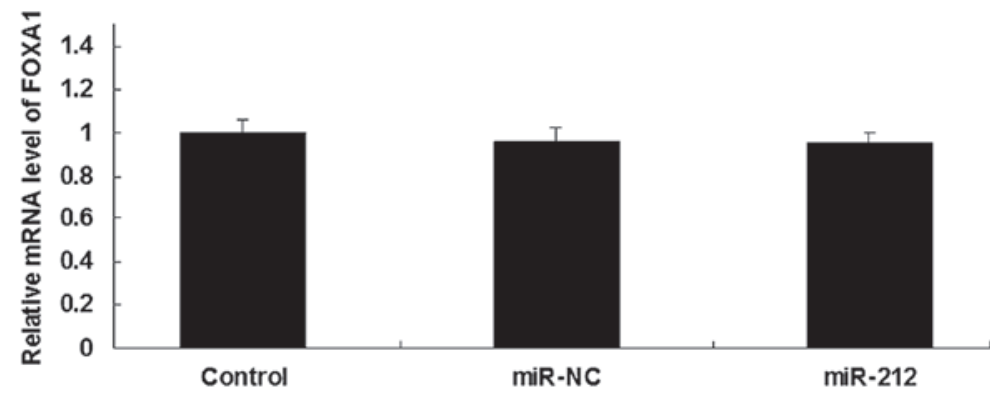

$\mathbf{B}$

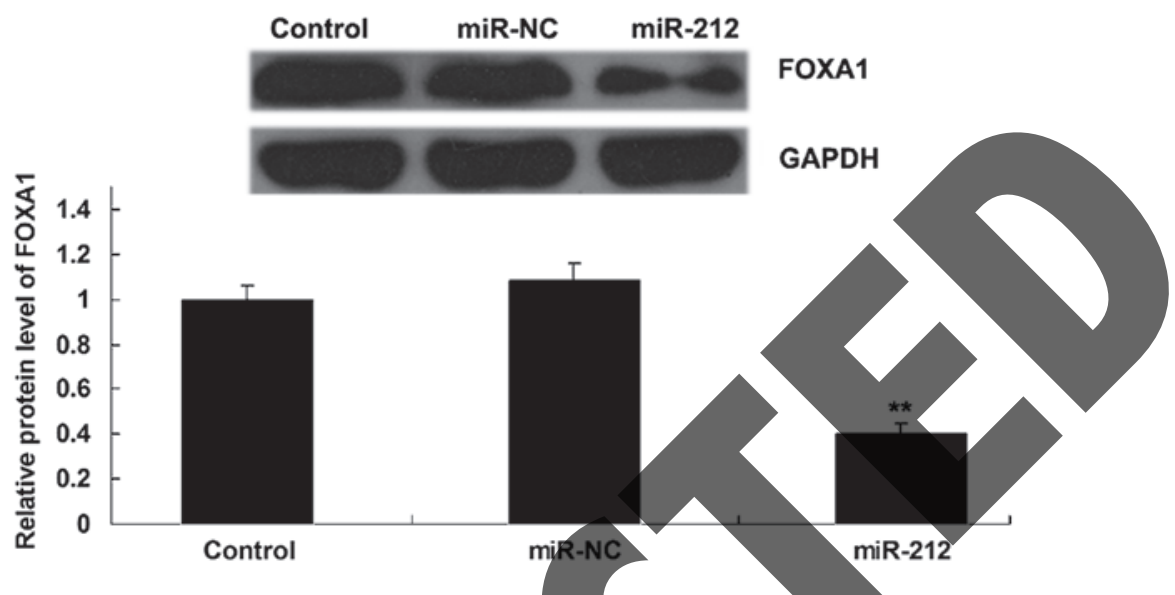

Figure 5. mRNA and protein expression levels of FOXA1 in intrahepatic cholangiocarcinoma QBC939 cells transfected with miR-212 mimics or miR-NC (A) Reverse transcription-quantitative polymerase chain reaction and (B) western blotting were used to determine the mRNA and protein expression levels of FOXA1 in QBC939 cells, respectively. Non-transfected QBC939 cells were used as a control. " P $<0.05$ vs. the control. FOXA1, forkhead box protein A1; miR-212, microRNA-212; miR-NC, microRNA-normal control; GAPDH, glyceraldehyde 3-phosphatedehydrogenase.

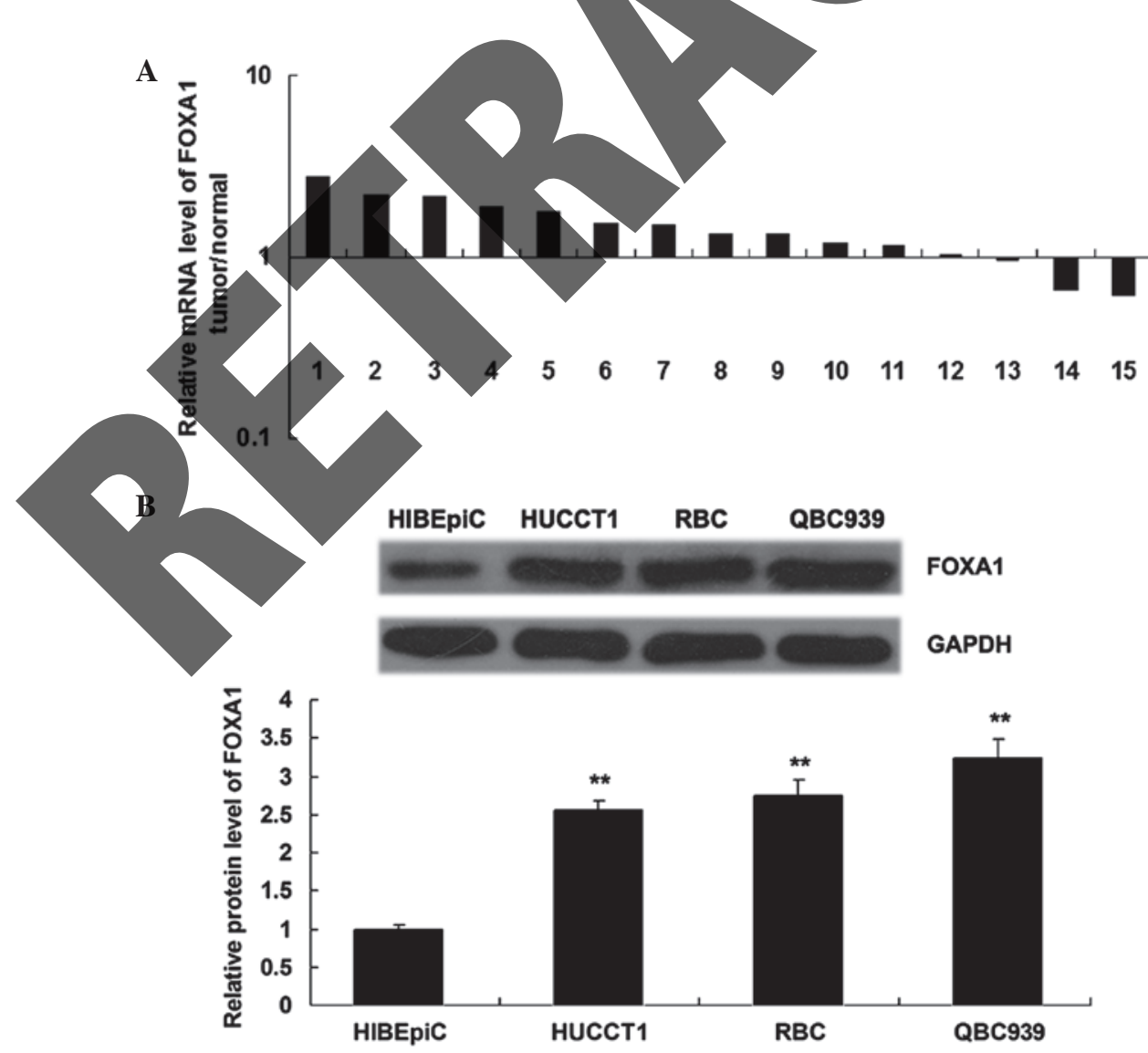

Figure 6. mRNA and protein expression levels of FOXA1 in intrahepatic cholangiocarcinoma (ICC) tissues and cell lines. (A) Reverse transcription-quantitative polymerase chain reaction was conducted to determine the mRNA expression levels of FOXA1 in ICC tissues, as compared with their matched adjacent normal tissues. (B) Western blotting was conducted to determine the protein expression levels of FOXA1 in ICC cell lines, as compared with HIBEpiC. ${ }^{* *} \mathrm{P}<0.05$ vs. HIBEpiC. FOXA1, forkhead box protein A1; HIBEpiC, human intrahepatic biliary epithelial cells; GAPDH, glyceraldehyde 3-phosphate dehydrogenase. 


\section{References}

1. Andersen JB: Molecular pathogenesis of intrahepatic cholangiocarcinoma. J Hepatobiliary Pancreat Sci 22: 101-113, 2015.

2. Sempoux C, Jibara G, Ward SC, Fan C, Qin L, Roayaie S, Fiel MI, Schwartz M and Thung SN: Intrahepatic cholangiocarcinoma: New insights in pathology. Semin Liver Dis 31: 49-60, 2011.

3. Sanada Y, Kawashita Y, Okada S, Azuma T and Matsuo S: Review to better understand the macroscopic subtypes and histogenesis of intrahepatic cholangiocarcinoma. World J Gastrointest Pathophysiol 5: 188-199, 2014.

4. Long XR, He Y, Huang C and Li J: MicroRNA-148a is silenced by hypermethylation and interacts with DNA methyltransferase 1 in hepatocellular carcinogenesis. Int J Oncol 44 1915-1922, 2014.

5. Tamagawa S, Beder LB, Hotomi M, Gunduz M, Yata K, Grenman R and Yamanaka N: Role of miR-200c/miR-141 in the regulation of epithelial-mesenchymal transition and migration in head and neck squamous cell carcinoma. Int J Mol Med 33: 879-886, 2014.

6. Wan X, Cheng Q, Peng R, Ma Z, Chen Z, Cao Y and Jiang B: ROCK1, a novel target of miR-145, promotes glioma cell invasion. Mol Med Rep 9: 1877-1882, 2014.

7. Liu X, Yan S, Pei C and Cui Y: Decreased microRNA-132 and its function in human non-small cell lung cancer. Mol Med Rep 11: 3601-3608, 2015.

8. Zheng K, Liu W, Liu Y, Jiang C and Qian Q: MicroRNA-133a suppresses colorectal cancer cell invasion by targeting Fascin1. Oncol Lett 9: 869-874, 2015

9. Chen P, Zeng M, Zhao Y and Fang X: Upregulation of Limk1 caused by microRNA-138 loss aggravates the metastasis of ovarian cancer by activation of Limk1/cofilin signaling. Oncol Rep 32: 2070-2076, 2014.

10. Tavolaro S, Colombo T, Chiaretti S, Peragine N, Fulci V, Ricciardi MR, Messina M, Bonina S, Brugnoletti F, Marinelli M, et al: Increased chronic lymphocytic leukemia prolifer upon IgM stimulation is sustained by the upregulation miR-132 and miR-212. Genes Chromosomes-Cancer 222-234, 2015

11. Qi B, Liu SG, Qin XG, Yao WJ, Lu JG, Guo L, Wang TY Li HC and Zhao BS: Overregulation of microRNA-212 in the poor prognosis of esophageal cancer patients. Genet Mol Res 13: 7800-7807, 2014.

12. Jiang X, Chen X, Chen L, Ma Y, Zhou L, Qi Q, Liu Y, Zhan Luo J and Zhou X: Upregulation of the miR-212/132 cluster suppresses proliferation of human lung cancer cells. Oncol Rep 33: 705-712, 2015.

13. Liang X, Zeng J, Wang L, Fang M, Wang Q, Zhao M, Xu X, Liu Z, Li W, Liu S, et al: Histone demethylase retinoblastoma binding protein 2 is overexpressed in hepatocellular carcinoma and negatively regulated by hsa-miR-212. PLoS One 8: e69784, 2013.

14. Ma C, Nong K, Wu B, Dong B, Bai Y, Zhu H, Wang W, Huang X, Yuan Z and Ai K: miR-212 promotes pancreatic cancer cell growth and invasion by targeting the hedgehog signaling pathway receptor patched-1. J Exp Clin Cancer Res 33: 54, 2014

15. Livak KJ and Schmittgen TD? Analysis of relative gene expression data using real-time quantitative PCR and the 2(-Delta Delta C(T)) Method. Methods 25: 402-408, 2001.

16. Fabbri M, Calore F, Paone A, Galli R and Calin GA: Epigenetic regulation of miRNAs in cancer. Adv Exp Med Biol 754: 137-148, 2013.

17. Bouyssou JM, Manier S, Huynh D, Issa S, Roccaro AM and Ghobrial IM: Regulation of microRNAs in cancer metastasis. Biochim Biophys Acta 1845: 255-265, 2014.

18. Wen KC, Sung PL, Yen MS, Chuang CM, Liou WS and Wang PH: MicroRNAs regulate several functions of normal tissues and malignancies. Taiwan J Obstet Gynecol 52: 465-469, 2013.
19. John K, Wu J, Lee BW and Farah CS: MicroRNAs in head and neck cancer. Int J Dent 2013: 650218, 2013.

20. Yamasaki S: Intrahepatic cholangiocarcinoma: Macroscopic type and stage classification. J Hepatobiliary Pancreat Surg 10: 288-291, 2003.

21. Zhao XQ, Ma HX, Su MS and He L: Osteopontin promoter polymorphisms at locus -443 are associated with metastasis and poor prognosis of human intrahepatic cholangiocarcinoma in Chinese population. Int J Clin Exp Pathol 7: 6914-6921, 2014.

22. Park JK, Henry JC, Jiang J, Esau C, Gusev Y, Lerner MR, Postier RG, Brackett DJ and Schmittgen TD: miR-132 and miR-212 are increased in pancreatic cancer and target the retinoblastoma tumor suppressor. Biochem Biophys Res Commun 406: 518-523, 2011

23. Schultz NA, Andersen KK, Roslind A, Willenbrock H, Wøjdemann $M$ and Johansen JS: Prognostic microRNAs in cancer tissue from patients operated for pancreatic cancer-five microRNAs in a prognostic index. World J Surg 36: 2699-2707, 2012.

24. Scapoli L, Palmieri A, Lo Muzio L, Pezzetti F, Rubini C, Girardi A, Farinella F, Mazzotta $M$ and Carinci F: MicroRNA expression profiling of oral carcinoma identifies new markers of tumor progression. Int J Immunopathol Pharmacol 23:

5. Wada R, Akiyama Y, Hashimoto Y, Fukamachi H and Yuasa Y: miR-212 is downregulated and suppresses methyl-CpG-binding protein MeCP2 in human gastric cancer. Int J Cancer 127: 1106-1114, 2010

26. Incoronato M, Urso L, Portela A, Laukkanen MO, Soini Y, Quintavalle C, Keller S, Esteller $M$ and Condorelli $G$ : Epigenetic regulation of miR-212 expression in lung cancer. PLoS One 6: e27722, 2011.

ian X, Samadani U, Porcella A and Costa RH: Decreased expression of hepatocyte nuclear factor 3 alpha during the ute-phase response influences transthyretin gene transcription. Mol Cell Biol 15: 1364-1376, 1995.

28. Hsiang CH, Marten NW and Straus DS: Upstream region of rat serum albumin gene promoter contributes to promoter activity: Presence of functional binding site for hepatocyte nuclear factor-3. Biochem J 338: 241-249, 1999.

9. Guzmán C,Benet M,Pisonero-VaqueroS,Moya M,García-Mediavilla MV, Martínez-Chantar ML, González-Gallego J, Castell JV, Sánchez-Campos S and Jover R: The human liver fatty acid binding protein (FABP1) gene is activated by FOXA1 and PPARalpha; and repressed by C/EBPalpha: Implications in FABP1 down-regulation in nonalcoholic fatty liver disease. Biochim Biophys Acta 1831: 803-818, 2013.

30. Horimoto Y, Arakawa A, Harada-Shoji N, Sonoue H, Yoshida Y, Himuro T, Igari F, Tokuda E, Mamat O, Tanabe M, et al: Low FOXA1 expression predicts good response to neo-adjuvant chemotherapy resulting in good outcomes for luminal HER2-negative breast cancer cases. Br J Cancer 112: 345-351, 2015.

31. Zhang Y and Tong T: FOXA1 antagonizes EZH2-mediated CDKN2A repression in carcinogenesis. Biochem Biophys Res Commun 453: 172-178, 2014

32. Yu W, Qiao Y, Tang X, Ma L, Wang Y, Zhang X, Weng W, Pan Q, Yu Y, Sun F and Wang J: Tumor suppressor long non-coding RNA, MT1DP is negatively regulated by YAP and Runx2 to inhibit FoxA1 in liver cancer cells. Cell Signal 26: 2961-2968, 2014

33. Zhao Y and Li Z: Interplay of estrogen receptors and FOXA factors in the liver cancer. Mol Cell Endocrinol: S0303-S7207, 2015. 